\title{
Generation of Ultralow Jitter Optical Pulses Using Optoelectronic Oscillators With Time-Lens Soliton-Assisted Compression
}

\author{
Yanne Kouomou Chembo, Abdelhamid Hmima, Pierre-Ambroise Lacourt, Laurent Larger, and \\ John M. Dudley, Fellow, OSA
}

\begin{abstract}
In this paper, we propose a new approach for the generation of ultralow jitter optical pulses using optoelectronic microwave oscillators. The short pulses are obtained through time-lens soliton-assisted compression of sinusoidally modulated prepulses, which are self-started from a conventional single-loop optoelectronic oscillator. The inherent ultralow phase noise of optoelectronic oscillators is converted into ultralow timing jitter for the generated pulses. We provide a time-domain model for the slowly varying amplitudes of the microwave and optical oscillations, and our analytical study is confirmed by numerical simulations and experimental measurements. We demonstrate the generation of 4.1 ps pulses along with a microwave whose phase noise is $-140 \mathrm{dBc} / \mathrm{Hz}$ at $10 \mathrm{kHz}$ from the $10 \mathrm{GHz}$ carrier, with $2.7 \mathrm{fs}$ jitter in the 1-10 kHz frequency band.
\end{abstract}

Index Terms-Microwave generation, nonlinear optics, nonlinear oscillators, optical pulse compression, optoelectronic devices, phase noise, timing jitter.

\section{INTRODUCTION}

$\mathbf{O}$ ptoelectronic oscillators (OEOs) are known to provide ultrapure microwaves with ultralow levels of phase noise [1], [2]. Their high quality factor is obtained through the storage of laser light energy in an optical fiber delay line instead of microwave storage in a high-finesse resonator. However, beside the generation of ultrapure microwaves in the RF range, OEO-like systems can also be used to generate optical pulses in the optical frequency range. The advantage of OEOs, in this case, is that their intrinsically low phase noise is equivalent to extremely low timing jitter for the generated pulses. Several architectures have been explored along that line, such as coupled mode-locked fiber laser OEOs [3], [4], electroabsorption OEOs [5], phase-modulation OEOs [6], or mutually coupled OEOs with intracavity fiber parametric amplification [7].

In this paper, we propose an efficient optoelectronic system that is able to generate short optical pulses with reduced timing

\footnotetext{
The authors are with the Optics Department, FEMTO-ST Institute, 25030 Besancon, France (e-mail: ckyanne@yahoo.fr; ahmima@univ-fcomte.fr; pierre-ambroise.lacourt@univ-fcomte.fr; $\quad$ laurent.larger@univ-fcomte.fr; john.dudley@univ-fcomte.fr).
}

jitter at a $10 \mathrm{GHz}$ rate. The architecture relies on the single-mode OEO, with two modulation branches: an intensity modulation for the formation of sinusoidal "prepulses" and a phase modulation to chirp them. The train of chirped prepulses is then strongly amplified by an erbium-doped fiber amplifier (EDFA) and subsequently launched into a single-mode optical fiber. Due to the combined effects of dispersion and self-phase modulation, the prepulses are optimally compressed with a compression factor that may be as high as 20 , following a scenario referred to as time-lens soliton-assisted compression [8]. This self-starting train of short pulses are converted back to an electrical signal, and then, they are filtered around the fundamental frequency $(=10 \mathrm{GHz})$ before being used to close the feedback loop through the two aforementioned modulation branches. The system therefore generates, at the same time, short, regularly spaced optical pulses in the optical domain, and an ultrapure microwave in the RF domain. With this architecture, the fiber delay line therefore plays two distinct and equally important roles: it stores optical energy as in conventional OEOs, and moreover, it compresses the sinusoidally modulated laser beam to a train of short pulses.

The outline of the paper is the following. In Section II, we present the system under study, and we build a related time-domain model for the optical and microwave variables in Section III. The stationary states and their stability are investigated in detail in Section IV, and using the split-step Fourier algorithm, we will numerically evidence the efficiency of the pulse compression. Section $V$ presents the experimental results, and the final section provides some concluding remarks.

\section{SYSTEM}

A schematic representation of the OEO under study is displayed in Fig. 1. The oscillation loop consists of the following elements.

1) A continuous-wave (CW) DFB semiconductor laser of optical power $P_{0}$, whose central wavelength is $\lambda_{0}=1550 \mathrm{~nm}$.

2) A wideband integrated optics $\mathrm{LiNbO}_{3}$ phase modulator (PM) characterized by a half-wave voltage $V_{\pi_{p}}=4 \mathrm{~V}$.

3) A wideband integrated optics $\mathrm{LiNbO}_{3}$ Mach-Zehnder (MZ) intensity modulator (IM) characterized by the half-wave voltages $V_{\pi_{\mathrm{dc}}}=6.6 \mathrm{~V}$ and $V_{\pi_{\mathrm{rf}}}=4.2 \mathrm{~V}$.

4) An EDFA of gain $G_{o}=24 \mathrm{~dB}$.

5) An optical filter (bandwidth $\sim 1 \mathrm{~nm}$ around $\lambda_{0}$ ) in order to reject the amplified spontaneous emission noise of the 


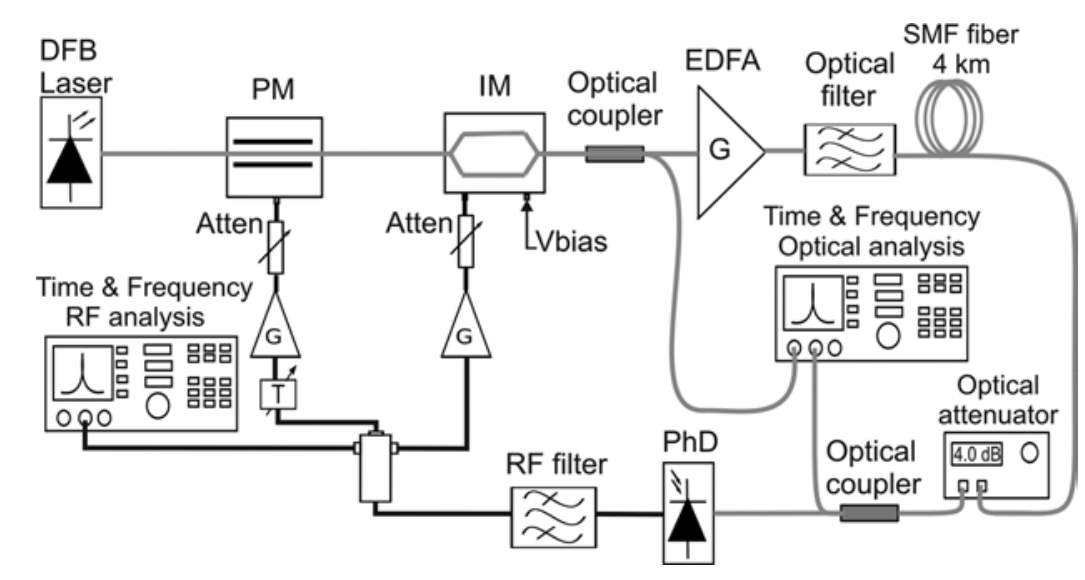

Fig. 1. Experimental setup for the optoelectronic pulse generator. The gray line represents the optical path and the black line represents the electrical path.

EDFA generated far from the central wavelength of the laser diode.

6) A thermalized single-mode fiber (SMF) of length $L=$ $4 \mathrm{~km}$, whose main characteristics are, respectively, the following: the group velocity of the laser light $v_{g}=1 / \beta_{1}=$ $c / n_{g}$ ( $c$ being the velocity of light in vacuum and $n_{g}=1.5$ being the group refractive index); the group velocity dispersion (GVD) parameter $\beta_{2}=-20 \mathrm{ps}^{2} / \mathrm{km}$; the linear fiber loss parameter $\alpha=0.2 \mathrm{~dB} / \mathrm{km}$ (or equivalently, exponential power decay factor of $\left.0.046 \mathrm{~km}^{-1}\right)$; and the nonlinear self-phase modulation (SPM) parameter $\gamma=$ $1.1 \mathrm{~W}^{-1} / \mathrm{km}$. This fiber delay line performs a time delay of $T_{D}=L / v_{g}=20 \mu \mathrm{s}$ on the microwave signal carried by the optical beam, and generates ring-cavity modes whose free spectral range is $\Omega_{T} / 2 \pi=1 / T_{D}=50 \mathrm{kHz}$.

7) An optical attenuator in order to reduce the optical power below the upper limit tolerated by the subsequent optoelectronic components.

8) A fast photodiode with a conversion factor $S=20 \mathrm{~V} / \mathrm{W}$ and bandwidth 0-18 GHz.

9) A narrowband microwave RF filter intended to select the frequency range for the amplified modes; its central frequency is $\Omega_{0} / 2 \pi=F_{\text {osc }}=10 \mathrm{GHz}$ and the $-3 \mathrm{~dB}$ bandwidth is $\Delta \Omega / 2 \pi=50 \mathrm{MHz}$, so that the related quality factor is $Q=\Omega_{0} / \Delta \Omega=200$.

10) Two microwave amplifiers with gain $G_{e}$ are required to close the loop to the phase and IMs. The corresponding RF signals may be attenuated in order to either vary the overall feedback gain (intensity modulation branch) or the strength of the chirp (phase modulation). A variable time-delay is also introduced in the phase modulation branch in order to control the phase shift between the two modulating signals.

The main difference between this pulse generator and the classical single-loop OEO is twofold: first, there is a phase modulation cascaded with intensity modulation, and second, an EDFA is included in the loop to strongly amplify the optical field in order to induce significant nonlinear effects in the fiber delay line.

\section{MODEL}

The dynamics of this system is defined by two variables. The first variable is the electric field $E(z, t)$ of the optical signal in the fiber, which depends on the time $t$ and the longitudinal dimension $z$ of the fiber (such that $0 \leq z \leq L$ ). The second variable is the input voltage $V(t)$ of the MZ modulator, which is only time dependent. All the typical distances of the system can be neglected relative to the fiber length, except when they induce qualitatively significant phase shifts.

Instead of working directly with the real variables $E(z, t)$ and $V(t)$, we use their complex slowly varying amplitudes (SVAs) $\mathcal{E}(z, t)=|\mathcal{E}(z, t)| e^{i \varphi(z, t)}$ and $\mathcal{V}(t)=|\mathcal{V}(t)| e^{i \psi(t)}$ defined by

$$
\begin{aligned}
E(z, t) & =\frac{1}{2} \mathcal{E}(z, t) e^{i \omega_{0} t}+\frac{1}{2} \mathcal{E}^{*}(z, t) e^{-i \omega_{0} t} \\
V(t) & =\frac{1}{2} \mathcal{V}(t) e^{i \Omega_{0} t}+\frac{1}{2} \mathcal{V}^{*}(t) e^{-i \Omega_{0} t}
\end{aligned}
$$

where $\omega_{0}$ and $\Omega_{0}$ are the angular frequencies associated with the $1550 \mathrm{~nm}$ IR laser beam and the $10 \mathrm{GHz}$ microwave signal, respectively.

The SVA of the optical beam at the output of the laser diode simply reads $\mathcal{E}_{\mathrm{cw}}=\sqrt{P_{0}}$. This beam is phase and amplitude modulated with a driving RF signal $V(t)=|\mathcal{V}(t)| \cos \left[\Omega_{0} t+\right.$ $\psi(t)]$, and then amplified with the EDFA: the SVA of the optical field at the input of the optical fiber is therefore

$$
\begin{array}{r}
\mathcal{E}(0, t)=\sqrt{\kappa_{o} G_{o} P_{0}} \cos \left\{\frac{\pi}{2} \frac{|\mathcal{V}(t)|}{V_{\pi_{\mathrm{rf}}}} \cos \left[\Omega_{0} t+\psi(t)\right]+\frac{\pi}{2} \frac{V_{B}}{V_{\pi_{\mathrm{dc}}}}\right\} \\
\times \exp \left\{i \pi \frac{\varrho|\mathcal{V}(t)|}{V_{\pi_{p}}} \cos \left[\Omega_{0} t+\psi(t)+\Delta \theta\right]\right\}
\end{array}
$$

where $\kappa_{o}$ synthetically gathers all the optical losses between the output of the laser and the input of the optical fiber, $V_{B}$ is the bias voltage of the MZ modulator, $\varrho$ is a dimensionless parameter measuring the amplitude ratio between the phase and intensity electro-optic modulations, while $\Delta \theta$ is the time-delay-induced phase shift between the phase and intensity modulations. We have assumed that the optical filtering for noise rejection is wide 
enough in order not to induce a dynamics of the optical field in the frequency range of interest.

The train of prepulses whose initial temporal profile is defined in (2) then travels through the fiber, and its dynamics is governed by the following propagation equation:

$$
\begin{aligned}
\frac{\partial \mathcal{E}(z, t)}{\partial z}=-\frac{\alpha}{2} \mathcal{E}(z, t)-\beta_{1} \frac{\partial \mathcal{E}(z, t)}{\partial t} & -i \frac{\beta_{2}}{2} \frac{\partial^{2} \mathcal{E}(z, t)}{\partial t^{2}} \\
& +i \gamma|\mathcal{E}(z, t)|^{2} \mathcal{E}(z, t)
\end{aligned}
$$

from which we can obtain the output train of pulses through $\mathcal{E}(L, t)$, knowing $\mathcal{E}(0, t)$ from (2). The previous equation can be reduced to a nonlinear Schrödinger equation (NLSE) with losses if rewritten in the moving frame.

The equation for the dynamics of the microwave signal at the input of the $\mathrm{MZ}$ is [9]

$V(t)+\frac{1}{\Delta \Omega} \frac{d}{d t} V(t)+\frac{\Omega_{0}^{2}}{\Delta \Omega} \int_{t_{0}}^{t} V(s) d s=\kappa_{o}^{\prime} \kappa_{e} G_{e} S|\mathcal{E}(L, t)|^{2}$.

On the other hand, $G_{e}$ is the electrical gain in the intensity modulation branch (the gain is then $\varrho G_{e}$ in the phase modulation branch), $\kappa_{e}$ is the overall electrical losses in either modulation branch, while $\kappa_{o}^{\prime}$ is the optical attenuation between the output of the optical fiber and the input of the photodiode. This equation therefore rules the conversion of the output optical power $|\mathcal{E}(L, t)|^{2}$ into a microwave voltage by the photodetector and its subsequent filtering around $\Omega_{0}$. This optical power can be spectrally decomposed as

$$
|\mathcal{E}(L, t)|^{2}=\frac{1}{2} \mathcal{C}_{0}(t)+\sum_{n=1}^{+\infty}\left\{\frac{1}{2} \mathcal{C}_{n}(t) e^{\mathrm{in} \Omega_{0} t}+\text { c.c. }\right\}
$$

where "c.c." stands for the complex conjugate of the preceding term.

The mathematical determination of the slowly varying Fourier components $\mathcal{C}_{n}(t)$ is not straightforward, as the definition of a slowly varying variable requires a rigorous definition of the time scales involved in the global dynamics under study. In our case, and according to (5), it means that the output power of the fiber has a fast time scale associated with the microwave frequency $\Omega_{0}$, and possibly a much slower time scale attached to $\mathcal{C}_{n}(t)$. We formally introduce the fast time scale under the dimensionless form $\tau=\Omega_{0} t$ for mathematical conciseness, then the slowly varying components $\mathcal{C}_{n}(t)$ can be computed using the Fourier scalar product

$$
\begin{aligned}
\mathcal{C}_{n}(t) & =\frac{1}{\pi} \int_{0}^{2 \pi}|\mathcal{E}(L, t, \tau)|^{2} e^{-\mathrm{in} \tau} d \tau \\
& \equiv\left\langle\left. e^{\mathrm{in} \Omega_{0} t}|| \mathcal{E}(L, t)\right|^{2}\right\rangle \cdot
\end{aligned}
$$

Note that in the synthetic bracket formulation, the fast time scale $\tau$ is implicit. Due to the resonant nature of the RF filter of the central frequency $\Omega_{0}$, only the spectral component $\mathcal{C}_{1}(t)$ of the optical signal $|\mathcal{E}(L, t)|^{2}$ detected at the photodetector is allowed to travel through the RF branch. Hence, the bandwidth of the photodetector should imperatively pass the fundamental frequency of the incoming pulse train, which is precisely $F_{\text {osc }}=$
$10 \mathrm{GHz}$ in our case. Therefore, assuming $\Omega_{T} \ll \Delta \Omega \ll \Omega_{0}$, the microwave envelope at the input of the MZ obeys [9]

$$
\frac{d \mathcal{V}(t)}{d t}=-\frac{\Delta \Omega^{\prime}}{2} e^{i \vartheta} \mathcal{V}(t)+\frac{\Delta \Omega^{\prime}}{2} e^{i \vartheta} \kappa_{o}^{\prime} \kappa_{e} G_{e} S\left\langle\left. e^{i \Omega_{0} t}|| \mathcal{E}(L, t)\right|^{2}\right\rangle
$$

The filter parameters of this equation are, respectively, the reduced bandwidth and the filter-induced phase shift defined as

$$
\Delta \Omega^{\prime}=\frac{\Delta \Omega}{\sqrt{1+[1 /(2 Q)]^{2}}} \text { and } \vartheta=\arctan \left[\frac{1}{2 Q}\right]
$$

where $Q=\Omega_{0} / \Delta \Omega$ is the quality factor of the RF filter. Earlier works have shown that the two approximations $\Delta \Omega^{\prime} \simeq \Delta \Omega$ and $\vartheta \simeq 0$ are sufficient for the study of the deterministic dynamics when $Q>100$ [9], [10]; however, only the first approximation is valid for the study of the stochastic dynamics (phase noise), as even a very small $\vartheta \simeq 1 /(2 Q)$ does play an important role in the presence of multiplicative noise [11]. We will then use these approximations, depending on the deterministic or stochastic nature of the analysis.

The model is therefore made up of two coupled evolution equations, namely, (3), whose temporal initial condition is (2), and (7). These equations, respectively, rule the dynamics of the optical field along the optical fiber and the microwave signal generated in the RF branch. The next section discusses the stationary states and their stability.

\section{STATIONARY States AND TheIR Stability}

\section{A. Weak-Signal Regime}

When the signal power circulating in the loop is weak, GVD and SPM are not relevant because their characteristic lengths (the dispersion length $L_{D}=T_{\text {pulse }}^{2} /\left|\beta_{2}\right|$ and the nonlinear length $L_{\mathrm{NL}}=1 /\left(\gamma|\mathcal{E}|_{\text {peak }}^{2}\right)$, respectively) are significantly greater than the delay line length $L$. This is due to the fact that $T_{\text {pulse }}$ is too large ( $\left.\sim 50 \mathrm{ps}\right)$ and $|\mathcal{E}|_{\text {peak }}^{2}$ is too weak. Then, as far as the fiber is concerned, we can simply consider attenuation and time-delay effects, leading to $|\mathcal{E}(L, t)|^{2}=e^{-\alpha L}\left|\mathcal{E}\left(0, t-T_{D}\right)\right|^{2}$, where $\alpha$ is the linear attenuation parameter. Hence, using (2), it can be shown that (7) may be rewritten as

$$
\begin{aligned}
\frac{d \mathcal{V}(t)}{d t}=-\frac{\Delta \Omega}{2} \mathcal{V}(t)-\Delta & \Omega \Gamma e^{-i \Omega_{0} T_{D} \mathrm{~J}_{1}} \\
\times & {\left[\pi \frac{\left|\mathcal{V}\left(t-T_{D}\right)\right|}{V_{\pi_{\mathrm{rf}}}}\right] \mathcal{V}\left(t-T_{D}\right) }
\end{aligned}
$$

where

$$
\Gamma=\frac{\pi \kappa_{o} \kappa_{o}^{\prime} \kappa_{e} G_{o} G_{e} S P_{0} e^{-\alpha L}}{2 V_{\pi_{\mathrm{rf}}}} \sin \left[\pi \frac{V_{B}}{V_{\pi_{\mathrm{dc}}}}\right]
$$

can be defined as the overall loop gain of the oscillator, while $\Omega_{0} T_{D}$ is the round-trip phase shift of the microwave and $\mathrm{J}_{c_{1}}$ is the Bessel-cardinal function defined as $\mathrm{Jc}_{1}(x)=\mathrm{J}_{1}(x) / x$.

This equation is identical to the one describing the classical single-loop OEO [9], [10]. The trivial equilibrium point $\mathcal{V}(t)=$ 0 and $\mathcal{E}(z, t)=0$ is stable for $|\Gamma|<1$, while for $1<|\Gamma|<$ 2.31 , a stable microwave of fixed amplitude and phase is generated, along with a sinusoidally modulated laser beam in the optical output. 
However, this equation loses validity well before the critical value $\Gamma_{\mathrm{cr}}=2.31$, because in the optical section, the signal is amplified by the EDFA, and $L_{D}$ and $L_{\mathrm{NL}}$ are significantly shortened so that the combination of GVD and SPM effects invalidates the simple hypothesis $|\mathcal{E}(L, t)|^{2}=e^{-\alpha L}\left|\mathcal{E}\left(0, t-T_{D}\right)\right|^{2}$. Therefore, (9) is an approximation, which is only valid under threshold or when the overall gain $|\Gamma|$ is only slightly greater than 1 .

\section{B. Strong-Signal Regime}

As the overall gain is increased, the optical signal amplified by the EDFA becomes powerful enough to generate combined GVD and SPM effects in the fiber. More specifically, the $\cos ^{2}$ temporal profile of the modulated laser beam can be considered as a train of prepulses, whose full-width at halfmaximum (FWHM) duration is approximately $50 \mathrm{ps}$, i.e., half the period $T_{\text {osc }}=2 \pi / \Omega_{0}=100 \mathrm{ps}$ of the modulating microwave signal. Time-lens soliton-assisted compression consists of chirping these prepulses in the sign opposite to the one of dispersion, so that as they travel, they are, at the same time, linearly compressed by chirp compensation and nonlinearly compressed by solitonic effects induced by GVD and SPM [8]. At the output of the fiber, the initial $50 \mathrm{ps}$ prepulses can be compressed down to few picoseconds, and the ultralow phase noise of the OEOs is here converted in ultralow temporal jitter between the pulses.

For the sake of simplicity, let us introduce the nonlinear operator $\hat{\mathbf{C}}$, here referred to as compression operator

$$
\mathcal{E}(L, t)=\hat{\mathbf{C}} \mathcal{E}\left(0, t-T_{D}\right) .
$$

This operator is, in fact, the formal solution of the propagation equation explicitly represented in (3), and transforms a train of prepulses into a train of shorter compressed pulses. Here, we restrict ourselves to time slots of duration $\Delta T=100 \mathrm{ps}$ corresponding to the period of the $10 \mathrm{GHz}$ microwave, so that the compression operator describes how a single prepulse is compressed during its propagation in the fiber.

This interpretation of $\hat{\mathbf{C}}$ is consistent with the numerical algorithms for solving the propagation equation, where the propagation of only one prepulse is simulated. This hypothesis also assumes that the propagating pulses do not interact with each other. Therefore, $\hat{\mathbf{C}}$, which depends on the parameters (attenuation, GVD, and SPM) of the propagation equation and the input prepulse (temporal profile, chirp), is numerically determined by comparing the temporal profile of the output pulse with the one of the input prepulse.

As we have seen in the precedent section, when the gain is weak, this operator simply becomes proportional to the identity operator, following $\hat{\mathbf{C}} \simeq e^{-(\alpha L / 2)}, \hat{\mathbf{I}}$. In fact, the same relation holds during the transient rising-up process because in this case, the signal is weak, regardless of the value of the overall gain $\Gamma$. It is, however, naturally expected that for strong signals, the compression operator should significantly differ from identity.

From (2), it appears that the prepulses whose profile varies monotonously from a minimum equal to zero to a single max- imum are achieved for a bias voltage $V_{B}$ and a steady-state microwave amplitude $\left|\mathcal{V}_{0}\right|$ obeying

$$
\frac{\left|\mathcal{V}_{0}\right|}{V_{\pi_{\mathrm{rf}}}}=\frac{V_{B}}{V_{\pi_{\mathrm{dc}}}}=\frac{1}{2} .
$$

In this case, the input energy of a prepulse is

$$
W_{\text {in }}=\int_{0}^{T_{\mathrm{osc}}}|\mathcal{E}(0, t)|^{2} d t=\frac{1}{2} \kappa_{0} G_{0} P_{0} T_{\mathrm{osc}}
$$

while the output energy of the compressed pulse may simply be evaluated as $W_{\text {out }}=e^{-\alpha L} W_{\text {in. }}$. Hence, the peak power of a compressed output pulse of duration $\Delta T_{\text {out }}$ may be estimated as

$$
P_{\text {peak }}=\frac{\zeta W_{\text {out }}}{\Delta T_{\text {out }}}=\zeta \eta \kappa_{0} G_{0} P_{0} e^{-\alpha L}
$$

where the factor $\zeta$ is the energetic fraction of the input prepulse energy that is effectively compressed into a pulse (the remaining part $1-\zeta$ is lost into the so-called pedestal, which is the small temporal ripple around central peak), and the factor $\eta=$ $\Delta T_{\text {in }} / \Delta T_{\text {out }}$ is the compression factor of the pulse, measuring the efficiency of the time-lens soliton compression process. The previous equation also indicates that independent of the various compression regimes, the ratio $P_{\text {peak }} /(\zeta \eta)$ should remain constant.

We should now determine the spectral coefficient $\mathcal{C}_{1}(t)$ that is reinjected into the RF branch of the feedback loop. The high compression factors we are expecting enable to consider in first approximation that the train of compressed pulses at the output of the optical fiber are not significantly discernible from a train of Dirac peaks (see Fig. 3)

$$
|\mathcal{E}(L, t)|^{2} \simeq \zeta W_{\text {out }} \sum_{n=-\infty}^{+\infty} \delta\left[t-n T_{\text {osc }}\right]
$$

leading to [using the scaling property $\left.\delta(t)=\Omega_{0} \delta(\tau)\right]$

$$
\left\langle\left. e^{i \Omega_{0} t}|| \mathcal{E}(L, t)\right|^{2}\right\rangle=\frac{2 \zeta W_{\text {out }}}{T_{\text {osc }}}
$$

It can, in fact, be shown that as long as the pulsewidth is significantly shorter than that of the half-period of the microwave (=50 ps), the Dirac pulse train approximation is excellent. This is due to the fact that when the pulsewidth $\tau$ is taken into account, the right-hand side of (16) should be multiplied by a term approximately equal to $\operatorname{sinc}\left[\Omega_{0} \tau\right]$, where $\Omega_{0}$ is the angular frequency of the microwave. As long as $\tau \ll T_{\text {osc }} / 2=50 \mathrm{ps, \text {we }}$ have $\Omega_{0} \tau \rightarrow 0$, so that this sinc term can be safely equated to 1 : in this case, the Dirac peaks approximation is sufficiently accurate. Therefore, using the assumption of steady-state oscillation $(d \mathcal{V} / d t \equiv 0)$, the evolution (7) gives the following value for the amplitude of the microwave:

$$
\mathcal{V}_{0}=\zeta \kappa_{o} \kappa_{o}^{\prime} \kappa_{e} G_{o} G_{e} S P_{0} e^{-\alpha L} .
$$




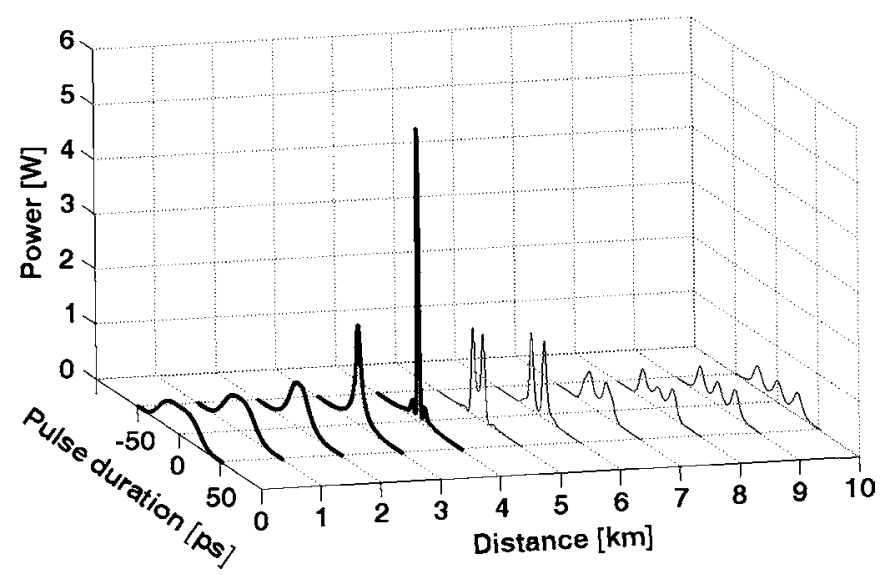

Fig. 2. Numerical simulation of the propagation of a pulse, evidencing timelens soliton-assisted compression. The parameters are $\mathcal{V}_{0}=2.1 \mathrm{~V}, V_{B}=$ $3.3 \mathrm{~V}, \varrho=1.5$, and $\Delta \theta=\pi$, and they fulfill (12). The input peak power at $z=0$ is $0.5 \mathrm{~W}$ while it is $5.3 \mathrm{~W}$ at $z=4 \mathrm{~km}$. The pulse is compressed from an FWHM of 50 ps down to $2.7 \mathrm{ps}$ with an energetic efficiency $\zeta=0.76$. The thick lines are the successive profiles of the pulse, which is finally extracted at $z=4 \mathrm{~km}$, while the thin lines display subsequent snapshots of the virtual pulse that would have propagated in a longer optical fiber. The initial energy of this pulse is $W_{\mathrm{in}}=25 \mathrm{pJ}$.

Using the definition of the overall feedback gain of (10) as well as (12), it can be shown that the previous equation provides the following optimal value for the feedback gain:

$$
\Gamma_{\text {opt }}=\frac{\pi}{4 \zeta}
$$

which thereby exclusively depends on the energetic efficiency $\zeta$ of the pulse compression. This optimal gain directly originates from a closure (or self-consistency) condition, which simultaneously assumes steady-state operation, high compression factor (quasi-Dirac output), and quasi-sinusoidal prepulse modulation. Since the condition $\Gamma>1$ is required for any oscillation to be triggered, it therefore arises that the condition $\zeta<\zeta_{\max }=\pi / 4$ should be absolutely fulfilled. In fact, this maximal value allowed for $\zeta$ to vary according to the operating point of the MZ modulator, but this analysis, however, surprisingly shows that imperfect pulse compression is necessary for a regular pulse train to be sustained.

We have implemented a split-step Fourier algorithm to simulate the propagation of the prepulses in the optical fiber. In Fig. 2, the propagation of a pulse is displayed in three dimensions. It can be observed that as the pulse propagates along the fiber, it is progressively compressed and the optimal compression is attained at the distance $z=L=4 \mathrm{~km}$. If the pulse is left to propagate any further, it breaks down into two, and then, three subpulses with significantly smaller peak power values.

The optimal dimension where the pulse is maximally compressed depends on all the parameters of the input pulse: power, profile, chirp, as well as on those of the fiber, i.e., GVD, SPM, and attenuation. A particularly suitable parameter to tune the optimal compression distance is the chirp intensity parameter $\varrho$, but it should be noted that the phase shift $\Delta \theta$ between the phase and intensity modulations also plays a critical role. In fact, we have presented here the compression regime for $\Delta \theta=\pi$, but
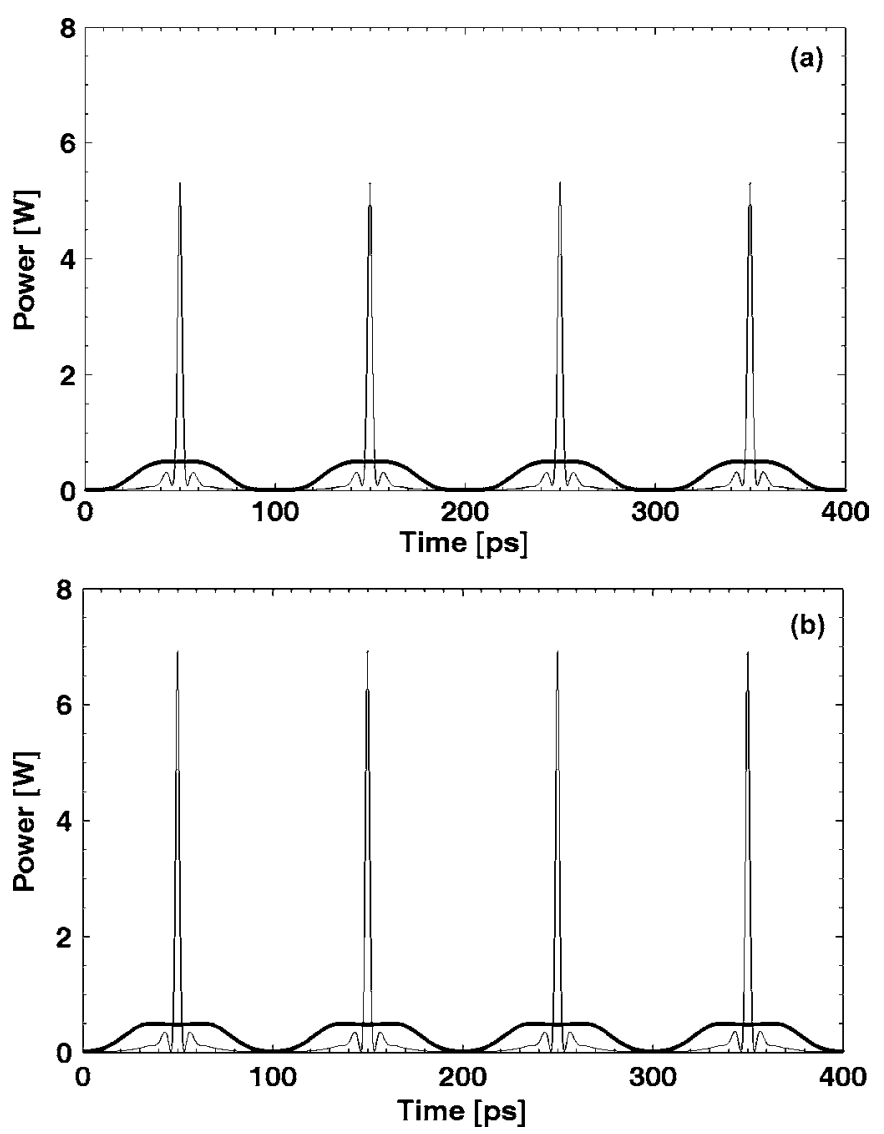

Fig. 3. Numerical simulation of the input and output pulse trains $|\mathcal{E}(z, t)|^{2}$ at $z=0$ (thick line) and $z=4 \mathrm{~km}$ (thin line), respectively. (a) Parameters are the same as those of Fig. 2 [case of (12)]. The pulse is compressed from 50 to $2.7 \mathrm{ps}(\eta=18.5)$, with a peak power of $5.3 \mathrm{~W}$ and an energetic efficiency $\zeta=0.76$. (b) Same parameters as Fig. 2, except $\mathcal{V}_{0}=2.15 \mathrm{~V}, V_{B}=2.4 \mathrm{~V}$, and $\varrho=1.6$. The pulse is compressed from 58 to $2.4 \mathrm{ps}(\eta=24.2)$, with a peak power of $7 \mathrm{~W}$ and an energetic efficiency $\zeta=0.72$. Note that this optimal regime yielding the maximal output peak power is only slightly detuned from case (a), fulfilling (12).

there is also a more robust compression regime for $\Delta \theta=\pi / 2$, even though the related compression factor is not as high.

Fig. 3 represents input train of prepulses as well as the simulated outputs. Fig. 3(a) shows how the four pulses (which are simulated simultaneously as a $400 \mathrm{ps}$ composite pulse) are compressed without interacting with each other, while Fig. 3(b) shows how a slight detuning from the maximal extinction condition may enable to enhance the pulse compression. In both cases, a limited efficiency in the compression process is evidenced as pedestals arise between the central peaks. However, our analytical study has demonstrated that the energetic efficiency coefficient is in the correct range for a pulse train to be steadily sustained in the system, as $\zeta<\zeta_{\max } \simeq 0.785$; moreover, a higher $\zeta$ would even be incompatible with pulse train generation. These values of $\zeta(\sim 0.7)$ also indicate that the value of the overall gain $\Gamma$ should be around $\Gamma_{\mathrm{opt}} \sim 1$ 1.1, i.e., only $10 \%$ above threshold. In fact, independent of the value of $\Gamma$ between 1 and a critical value $\Gamma_{\text {cr }}^{\prime}$ (which should be the counterpart of $\Gamma_{c r}=2.31$ in the single-loop OEO), an ultrapure microwave is generated at the RF output, but short pulses are not obtained at the optical output, unless the optimal conditions expressed in (12) and (18) are (at least approximately) fulfilled. 

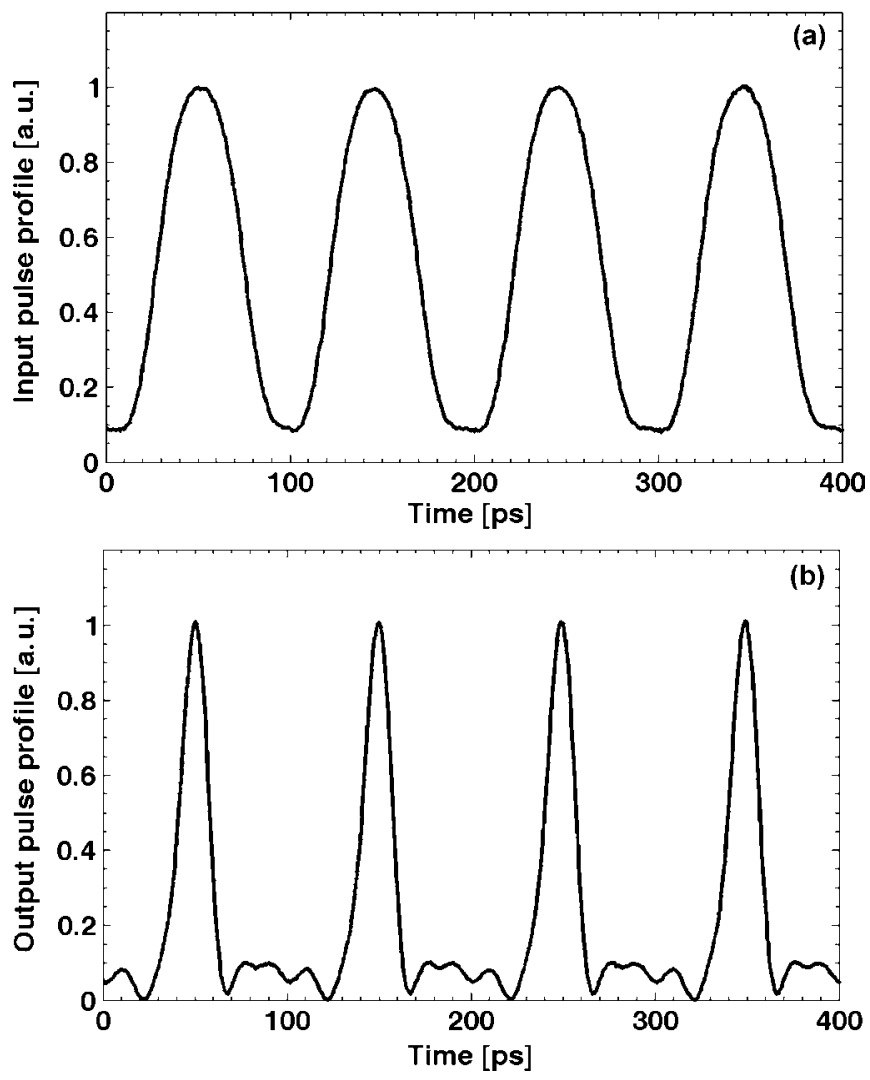

Fig. 4. Experimental results (oscilloscope display) showing the normalized temporal profiles of the input prepulse and output compressed pulse. The oscilloscope has a pulsewidth resolution of 15 ps. (a) Input pulse profile, with approximately a $50 \mathrm{ps}$ width. (b) Output pulse profile. In fact, the real pulses have a width, which is significantly shorter than the resolution of oscilloscope, so that the pulse train actually displays the impulse response of the oscilloscope, i.e., 15 ps.

\section{EXPERIMENTAL RESULTS}

\section{A. Pulse Compression}

At the experimental level, our task has been to monitor the input prepulses and output compressed pulses using a fast sampling oscilloscope with a wideband $(60 \mathrm{GHz})$ optical to electrical converter at its input. In Fig. 4, we have displayed the input and output normalized profiles of the pulses. Although the expected pulsewidth (few picoseconds) is smaller than the impulse response of the oscilloscope ( $15 \mathrm{ps}$ ), these time traces illustrate the qualitative effect of the time-lens soliton-assisted compression on the incoming prepulse train.

The width of the compressed pulses has been estimated more precisely using optical autocorrelation. The measured intensity correlation function shown in Fig. 5 is consistent with the qualitative form of the compressed pulse in the simulations shown in Fig. 3, presenting a central lobe whose FWHM is equal to $6.3 \mathrm{ps,}$ superimposed on a low amplitude pedestal. This result allows us to infer a central peak in the compressed pulse of 4.1 ps corresponding to a compression factor $\eta=12.2$.

This pulse-width is, however, higher than the one obtained from numerical simulation. This discrepancy is explained by the fact that a very small mismatch relative to the optimal compression parameters leads to a notable desgradation of the compression factor $\eta$. On the other hand, cross-polarization coupling effects have not been taken into account in the model, and they

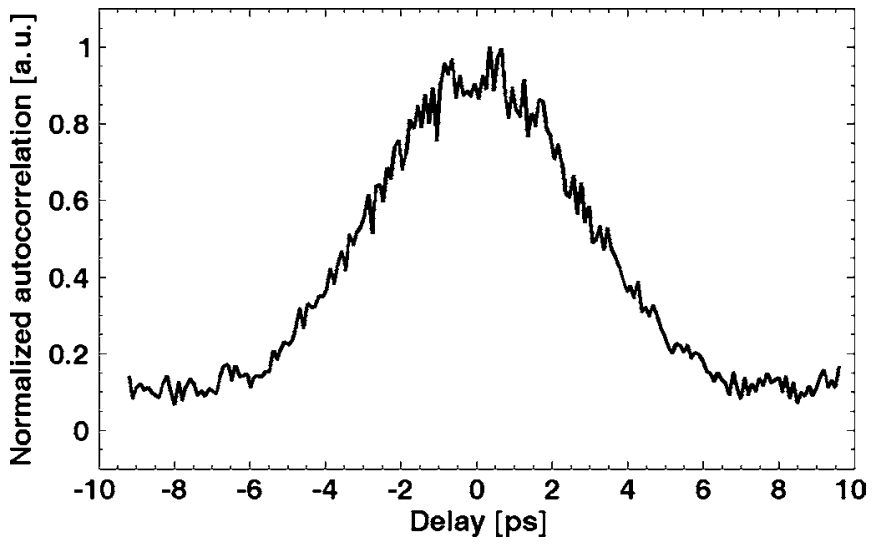

Fig. 5. Experimental autocorrelation plot. The FWHM of the plot is $6.3 \mathrm{ps}$, so that by assuming a deconvolution factor equal to 1.54, the FWHM for the pulses can be evaluated as $4.1 \mathrm{ps}$ and the corresponding compression factor $\eta=12.2$.

may also affect the efficiency of the compression process. The possibility of fine tuning of the parameters and a better controllability of the physical processes taking place in the fiber would allow values of the order of 20 for the compression factor.

As far as quality factor of the OEO is concerned, it is known that it can be estimated as $Q_{\mathrm{OEO}}=2 \pi F_{\mathrm{osc}} T_{D}=\Omega_{0} T_{D}$, where $F_{\text {osc }}$ is the microwave frequency and $T_{D}=n_{g} L / c$ the time-delay. Although there is no direct dependence on the nonlinearity in this equation, the dependence of oscillator $Q$ on fiber length and nonlinearity can indeed be understood using qualitative arguments. In fact, the fiber nonlinearity and length must be matched to the required power level to ensure appropriate nonlinear compression. Operating with a very high nonlinear fiber would allow operation at lower powers for the same nonlinear phase shift with the same fiber length. On the other hand, if high output power was an oscillator requirement, then highly nonlinear fiber would likely be detrimental to oscillator $Q$ as it could lead to very short optimal fiber length. The important point is that a careful consideration of required power levels and desired $Q$ should inform the selection of fiber nonlinearity and length.

\section{B. Phase Noise and Timing Jitter}

We have determined the phase noise $\mathcal{L}(f)$ of the output microwave using a customized phase noise measurement bench. Note that instrumental limitations preclude the use of direct oscilloscope-based characterization of this ultralow level of timing jitter. A theory for phase noise determination in OEOs has been proposed in [11], and globally, this theory holds for this OEO also. However, an explicit formula for the phase noise spectrum would require an explicit determination for the compression operator $\hat{\mathbf{C}}$, which is not available. Fig. 6 displays the result of our phase noise measurement, and it can be seen that for frequencies less than $10 \mathrm{kHz}$, there is an almost uniform $-30 \mathrm{~dB} /$ decade decrease: this is the signature of a dominant flicker noise in this frequency range. The noise floor is remarkably low, around $-140 \mathrm{dBc} / \mathrm{Hz}$ at $10 \mathrm{kHz}$ (with a phase noise floor of $-143 \mathrm{dBc} / \mathrm{Hz}$ around $20 \mathrm{kHz}$ ), thereby indicating an ultrahigh purity for the output microwave. 


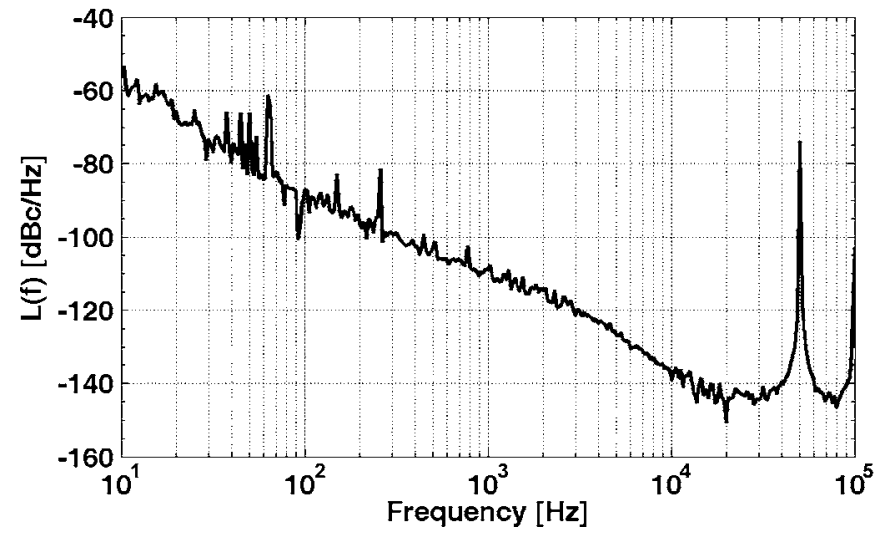

Fig. 6. Experimental phase noise spectrum of the microwave. The phase noise floor at $10 \mathrm{kHz}$ is around $-140 \mathrm{dBc} / \mathrm{Hz}$. The peak at $50 \mathrm{~Hz}$ (and its harmonics) originates from the mains supply, while the spurious peak at $50 \mathrm{kHz}$ is a delay mode peak corresponding to the round-trip frequency $1 / T_{D}$.

As explained in Section I, there is a relationship between phase noise and timing jitter: more precisely, a very low phase noise is equivalent to a very low timing jitter for the microwave. Knowing the phase noise spectrum $\mathcal{L}(f)$, the rms of this jitter can be determined as follows:

$$
\sigma=\frac{1}{2 \pi F_{\mathrm{osc}}} \sqrt{2 \int_{f_{l}}^{f_{u}} 10^{\mathcal{L}(f) / 10} d f}
$$

where $f_{l}$ and $f_{u}$ are, respectively, the lower and upper boundaries of the frequency range for which the jitter is computed. In our case, the experimental phase noise spectrum yields a value $\sigma=2.7 \mathrm{fs}$ in the $1-10 \mathrm{kHz}$ band. Such a precision for the microwave timing jitter also ensures a very high regularity for the output pulses, which may, therefore, be used in applications where a particularly high precision is required, such as in LIDAR technologies or optical sampling. It is important to note that the jitter is unequivocally bound to the phase noise of the oscillator, which depends on the $Q$-factor of the OEO. Therefore, if, for example, the fiber length decreases, then the $Q$-factor would also decrease, the phase noise would increase, as would also do the timing jitter. We also emphasize that if the timing jitter of the $10 \mathrm{GHz}$ microwave component observed in a long time span can be as low as $2.7 \mathrm{ps}$, then the locally observed pulse-to-pulse timing jitter, which does not contribute to OEO operation, is, in fact, much larger than that value.

\section{CONCLUSION}

In this paper, we have demonstrated a new optoelectronic architecture for the simultaneous generation of ultrapure microwaves and ultralow jitter optical pulses. We have proposed a theory to explain the generation of the self-starting pulse train, and our numerical simulations have been shown to be consistent with the experimentally observed phenomena. This dual generation is particularly useful as the microwave can directly play the role of the trigger signal in applications where optical pulses have to be synchronized to a compatible external source.
It may be also interesting to extend this pulse generation scheme with other types of OEOs, in particular, architectures that are able to cancel the spurious peaks (multiloop OEOs), or those that can be miniaturized (whispering-gallery mode OEOs). Along the same lines, special types of fibers can be used in order to engineer the fiber nonlinearity and/or dispersion to provide added flexibility in obtaining particular oscillator characteristics under specified conditions [6], [12], [13]. We anticipate that appropriate matching of fiber and source power requirements could lead to subpicosecond optical pulse generation with still an extremely low timing jitter.

\section{REFERENCES}

[1] X. S. Yao and L. Maleki, "Optoelectronic microwave oscillator," J. Opt. Soc. Amer. B, Opt. Phys., vol. 13, pp. 1725-1735, 1996.

[2] X. S. Yao and L. Maleki, "Optoelectronic oscillator for photonic systems," IEEE J. Quantum Electron., vol. 32, no. 7, pp. 1141-1149, Jul. 1996.

[3] X. S. Yao and L. Maleki, "Dual microwave and optical oscillator," Opt. Lett., vol. 22, pp. 1867-1869, 1997.

[4] N. Yu, E. Salik, and L. Maleki, "Ultralow-noise mode-locked laser with coupled optoelectronic oscillator configuration," Opt. Lett., vol. 30, pp. 1231-1233, 2005.

[5] J. Lasri, P. Devgan, R. Tang, and P. Kumar, "Self-starting optoelectronic oscillator for generating ultra-low jitter high-rate $(10 \mathrm{GHz}$ or higher) optical pulses," Opt. Exp., vol. 11, pp. 1430-1435, 2003.

[6] Y. Jiang, J.-L. Yu, H. Hu, W.-R. Wang, Y.-T. Wang, and E.-Z. Yang, "Phase-modulator-based optoelectronic oscillator for generating short optical pulse and microwave signal," Opt. Eng. Lett., vol. 46, pp. 090502-1-090502-3, 2007.

[7] D. Dahan, E. Shumakher, and G. Eisenstein, "Self-starting ultralow-jitter pulse source based on coupled optoelectronic oscillators with an intracavity fiber parametric amplifier," Opt. Lett., vol. 30, pp. 1623-1625, 2005.

[8] M. Hanna, P.-A. Lacourt, S. Poinsot, and J. M. Dudley, "Optical pulse generation using soliton-assisted time-lens compression," Opt. Exp., vol. 13, pp. 1743-1748, 2005.

[9] Y. K. Chembo, L. Larger, and P. Colet, "Nonlinear dynamics and spectral stability of optoelectronic microwave oscillators," IEEE $J$. Quantum Electron., vol. 44, no. 9, pp. 858-866, Apr. 2008.

[10] Y. K. Chembo, L. Larger, H. Tavernier, R. Bendoula, E. Rubiola, and P. Colet, "Dynamic instabilities of microwaves generated with optoelectronic oscillators," Opt. Lett., vol. 32, pp. 2571-2573, 2007.

[11] Y. K. Chembo, K. Volyanskiy, L. Larger, E. Rubiola, and P. Colet, "Determination of phase noise spectra in optoelectronic microwave oscillators: A Langevin approach," IEEE J. Quantum Electron., vol. 45, no. 2, pp. 178-186, Feb. 2009.

[12] J. Yu, "A simple and stable method to generate ultra-short optical pulse by using EAM and fibers," Opt. Commun., vol. 225, pp. 307-311, 2003.

[13] H. Hu, J. Yu, N. Bai, L. Zhang, A. Zhang, W. Wang, and E. Yang, "10 $\mathrm{GHz} 1.6$ ps optical pulse generation with 84 fs timing jitter," Electron. Lett., vol. 43, no. 22, pp. 1222 -1223, Oct. 2007. 